\title{
Normalidad y diferencia en la escuela: Diseño de un proyecto de investigación social desde el dilema político-epistemológico*
}

\author{
Normalcy and difference in schools: Social research project design \\ from the political-epistemological dilemma \\ Normalidade e a diferença na escola: Desenho de um projeto de pesquisa
social a partir do dilema político-epistemológico \\ Claudia Matus Cánovas, ${ }^{a}$ Andrés Haye Molina ${ }^{b}$ \\ aPontificia Universidad Católica de Chile, Santiago, Chile. \\ Teléfono: (56) 2 23545376. Correo electrónico: cmatusc@uc.cl

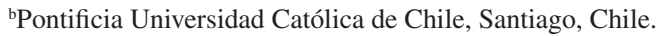 \\ Teléfono: (56) 2 23545315. Correo electrónico: ahaye@uc.cl
}

\begin{abstract}
RESUMEN
En este artículo se presenta una reflexión sobre la articulación entre lo político y lo epistemológico en una investigación enfocada sobre la producción de la normalidad y la diferencia en espacios escolares. Nuestra investigación asume que lo diferente no es un hecho natural ni una construcción aislada, sino que es una producción que se realiza en función de una normalidad imaginada y sostenida por instituciones, discursos y prácticas específicas que se pueden rastrear para comprender sus efectos y operaciones. La normalidad y la diferencia tienen por tanto un elemento eminentemente político, al igual que los estudios que parten de dichos discursos y prácticas para generar conocimiento sobre lo normal y lo diferente. En este artículo presentamos un proyecto de investigación que busca poner de manifiesto la producción de la normalidad para así repensar y transformar las prácticas escolares de la diferencia. Se reconstruye en detalle el problema de investigación y se describe su traducción a dispositivos metodológicos, a la luz de los dilemas derivados de la relación de lo epistemológico con lo político en cualquier investigación crítica, tal como los hemos enfrentado al elaborar nuestro proyecto.
\end{abstract}

Palabras clave: normalidad, diferencia, poder.

\section{ABSTRACT}

This article presents a reflection on the articulation between the political and epistemological dimensions of a research study focused on the production of normalcy and difference in school contexts. Our research undertakes that what we call "difference" is not a natural fact neither an isolated cultural construction. Rather, it is an ideological production in relation to an imagined normalcy which is fashioned through the workings of several institutions, discourses, and practices. As it is, these productions can be traced to understand their effects and operations. Normalcy and difference, then, are always political. In this article we present a research project that seeks to make visible the workings of the production of normalcy to rethink and transform those school practices about difference. We present the research problem in detail and then describe methodological aspects, emphasizing those political and epistemological dilemmas present in our project as a critical study.

Key words: normalcy, difference, power.

Artículo financiado por el Proyecto Anillos en Ciencias Sociales y Humanidades SOC1103 Normalidad, Diferencia y Educación, CONICYT, www.nde.cl. 


\section{RESUMO}

Apresenta-se uma reflexão sobre a articulação entre o político e o epistemológico em uma investigação focalizada na produção da normalidade e da diferença em espaços escolares. Assume-se que o diferente não é um fato natural nem uma construção isolada e, sim, uma produção que se realiza em função de uma normalidade imaginada e sustentada por instituições, discursos e práticas específicas que se podem rastrear para compreender seus efeitos e operações. A normalidade e a diferença têm, portanto, um elemento eminentemente político, assim como os estudos que partem de tais discursos e práticas para gerar conhecimento sobre o normal e o diferente. Apresenta-se um projeto de investigação que busca manifestar a produção da normalidade para, assim, repensar e transformar as práticas escolares da diferença. Reconstrói-se, detalhadamente, o problema de investigação e descreve-se sua tradução, à luz dos dilemas derivados da relação do epistemológico com o político em qualquer investigação crítica, tal como o temos enfrentado ao elaborar tal projeto.

Palavras chave: normalidade, diferença, poder.

\section{INTRODUCCIÓN}

En las últimas décadas ha habido un énfasis importante en la producción de políticas culturales de la diferencia en las escuelas y en las instituciones de educación superior. Instituciones de corte internacional (OEA, 1998; OECD, 2008; UNESCO, 1994, 2005) han destacado la importancia de pensar la formación de profesores y profesoras para unas salas de clases que cada vez estarán pobladas con estudiantes con distintas capacidades, provenientes de distintos países, etc. Esto abre un escenario en donde la diversidad y la diferencia se piensan como parte constitutiva de los contextos escolares.

En el caso particular de Chile se agregan don instancias legales que configuran los horizontes de las regulaciones de situaciones de discriminación dentro de la escuela: una de ellas es la Ley de Antidiscriminación (2012) y la reciente promulgada Ley de Inclusión Escolar (2015). Junto con esto, el Ministerio de Educación ha reforzado sus esfuerzos para orientar unas políticas más atingentes a las realidades de los establecimientos escolares en donde, a través de instrumentos como el Manual de Convivencia, se intenta resguardar y generar protocolos de prevención de situaciones no deseadas dentro del establecimiento.

A todos estos esfuerzos se les enmarca dentro de las iniciativas para impulsar políticas para la diversidad o inclusión y que como es lógico de esperar, tienen distintas formas de ser traducidas dependiendo de las posturas de cada establecimiento. Por ejemplo, en Chile, las formas de hacerse cargo de los temas de diversidad en las escuelas de formación de profesores y profesoras ha sido trabajado primordialmente a través de los discursos de discapacidad y necesidades educativas especiales (Infante \& Matus, 2009; Matus \& Infante, 2011). Esto ha generado que se sostenga una idea reducida y limitada de lo que anunciamos cada vez que hablamos de diversidad. Es así cómo se piensa que la "diversidad" es esta categoría que reúne a todas aquellas identidades que por alguna razón son pensadas como deficitarias o en necesidad de ser reparadas o compensadas. Por lo tanto, la diversidad así imaginada requiere hacer el ejercicio de localizar la diferencia en los cuerpos de sujetos particulares. También sugiere que la diferencia se observa, se diagnostica y se "mejora." Aun cuando para mucha gente esta forma de pensar la diferencia estaría bien informada para el caso de la discapacidad, el problema es aún mayor cuando esta lógica se extiende a "otras diferencias" y nos encontramos con discursos en donde se plantea que se necesitan herramientas especiales para trabajar con estudiantes peruanos, estudiantes pobres, estudiantes vulnerables, etc. En otras palabras, el peso "natural" que se le da a los temas de discapacidad y necesidades educativas especiales, como la forma obvia de justificar y enmar- 
car temas de diversidad en las escuelas, es problemática. Es problemática porque produce una idea limitada de cómo se construye y organiza la diferencia en los contextos escolares.

Es a partir de estas problemáticas que levantamos unas preguntas de investigación que proponen que la diferencia no es una construcción aislada ni natural, como si estuviese disponible para hacer uso de ella, sino que es una producción que se realiza en función de una normalidad imaginada y sostenida por instituciones, discursos y prácticas específicas que se pueden rastrear para comprender sus efectos y operaciones. En este caso la normalidad opera como una ideología (Davis, 2013) que encauza las formas en que presentamos argumentos para definir y diagnosticar al desviado, al que opera fuera de las normas de lo aceptado, valorado y deseado.

Para el caso de nuestra investigación, el insistir en investigar y documentar a aquellos grupos que son definidos y designados como diferentes, minoritarios, marginales, vulnerables solo sigue reproduciendo la causa de su marginalización. Por otro lado, se podría plantear que ya sabemos lo suficiente acerca de estos grupos y que aun así, no se ha recuperado el espacio político legítimo que les corresponde. Es por eso que en esta investigación, hacemos el ejercicio intelectual de producir conocimiento acerca de cómo la normalidad es producida y circulada en los espacios escolares.

Las preguntas de investigación que guían esta experiencia investigativa tienen que ver con ¿a propósito de qué normalidades es que los géneros, las sexualidades, las razas, las etnias, las clases sociales, son pensadas como productoras de sujetos diferentes? ¿Cuáles son las articulaciones que se hacen entre lo que imaginamos como normal y aquello que se desvía? Y en términos metodológicos ¿cómo se debiera registrar la normalidad? ¿Cuáles son las preguntas de investigación que debieran orientar una metodología para producir conocimiento acerca de la normalidad?

Estas preguntas tienen un carácter inalienablemente político en tanto remiten a ideologías y formas de pensar "lo natural" y "lo obvio" de las posiciones sociales y culturales de los sujetos. Sin duda que para avanzar en un conocimiento crítico de la diferencia es imperativo que se ponga en la escena aquello en relación a lo cual valoramos algo como diferente, raro, extraño. La indagación científica de vuelve así política en tanto cuestiona las condiciones de producción de las normalidades naturales y obvias. Esta politicidad epistemológica es el complejo contexto interno en el diseño del proyecto.

En el siguiente artículo queremos presentar dos componentes claves para el trabajo investigativo desarrollado. Como primer momento presentaremos el problema a investigar exponiendo algunos elementos teóricos claves para su formulación como también estadísticas nacionales que dan cuenta de la relevancia de exigir un ejercicio de producción de conocimiento como el llevado a cabo en esta investigación. En una segunda parte presentamos algunos elementos de cómo nos acercamos a contestar las preguntas de investigación, traduciendo las preguntas a decisiones metodológicas. Finalmente, discutiremos algunas dificultades generadas a partir de nuestro ejercicio, en términos del dilema entre lo político y lo epistemológico en la investigación.

\section{LA PRODUCCIÓN DEL PROBLEMA DE INVESTIGACIÓN}

Como mencionábamos al comienzo de este escrito, es importante considerar el énfasis que ha habido en las últimas décadas por generar unas políticas que vayan haciendo eco 
de demandas sociales acerca de la integración e inclusión de todos y todas en las escuelas. Es así como el Ministerio de Educación, a partir de sus esfuerzos en materia de reforma educativa, propone la creación de la coordinación de inclusión al interior de la Dirección de Educación General con el fin de promover unas políticas que tengan que ver con el tema de la diferencia y que tengan el impacto necesario para mostrar cambios al interior de los espacios escolares.

Como también se mencionaba en un principio, en el caso de Chile las políticas de diversidad se han orientado mayoritariamente a relevar el tema de la discapacidad y de las necesidades educativas especiales como centrales para este trabajo (Infante \& Matus, 2009). Sin embargo, esto ha producido ideas restringidas acerca de la construcción de la diferencia, que ha impactado las formas en que, por ejemplo, los programas de formación de profesores y profesoras han pensado la formación de sus estudiantes. Concretamente, si ponemos atención a aquellas políticas que se hacen cargo de los temas de diversidad en las escuelas, se observa que generalmente lo hacen desde el diseño e implementación de metodologías para organizar la sala de clases, evaluar a estudiantes poniendo especial atención a sus diferencias particulares o promoviendo más sensibilidad a las necesidades de "estos" estudiantes que son diagnosticados (Infante, 2007).

Cuando las políticas nacionales que piensan los temas de diversidad enfatizan las dimensiones administrativas y pedagógicas, promueven que los agentes escolares planteen los temas de diferencia de manera reduccionista y esencialista, aun cuando se busque reparar los efectos opresivos de estas diferencias. El supuesto que subyace a estas políticas es que los individuos son diferentes ya sea por raza, clase, capacidad, género, etc., y luego de aceptar este hecho, se puede comprender cómo medir, monitorear y controlar este hecho (Baez, 2004). Esta forma de razonar la diversidad produce la idea de que las políticas educativas deben tender a compensar a estos y estas estudiantes que son pensados como diferentes.

En nuestro proyecto, esto no es suficiente para promover cambios culturales y sociales en los contextos educativos. Dado que hemos asumido que la diferencia es solo posible y reconocible a propósito de una normalidad, nuestros supuestos están sostenidos en la idea de que la producción de conocimiento escolar, las prácticas sociales y las relaciones de poder se generan a partir de los sistemas aprendidos para razonar dimensiones tales como género, sexualidad, raza, discapacidad y clase social, lo que produce la marginación de algunos grupos, situación que queda expresada en el número creciente de prácticas de abuso y discriminación en las escuelas. La pregunta por la producción de la normalidad y la diferencia en la escuela obliga a cuestionar los supuestos en que se fundan las políticas públicas hacia la diversidad, que son a partir de las cuales se configuran los problemas escolares, y que tienen un impacto en cómo se organizan y priorizan los temas y formas de hacer investigación en estas áreas. Por ello, para definir el problema de este proyecto se ha decidido no poner la atención en la segregación misma del sistema educativo chileno, ni tampoco abordar las diferencias desde la "brecha académica" entre distintos establecimientos, sino que más bien en explorar cómo las formas de razonar sobre quiénes somos y las posiciones sociales y culturales que ocupamos en la sociedad, son aprendidas y enseñadas en la escuela.

En este contexto, los fundamentos empíricos de nuestra investigación pertenecen a dos grupos de datos relevantes. Primero, las estadísticas que indican altos porcentajes de situaciones en las que predominan los estereotipos y las situaciones de prejuicio y 
discriminación en el sistema escolar. Segundo, las políticas educativas que han emergido con el fin de regular y sancionar aquellos comportamientos que no promueven una convivencia sana y de calidad en los establecimientos educacionales. En referencia al primer grupo de antecedentes, las estadísticas de los últimos años en Chile, en relación a temas como género, sexualidad, VIH/SIDA, familia, entre otros, indican que hay una porción importante de nuestra población que discrimina (CEP, 1995, 2012; UNICEF 2011). Y más importante aún, es que estas cifras indican que los/las estudiantes, a medida que avanzan en la trayectoria escolar, van "convirtiéndose" en sujetos que reproducen ideas esencialistas en relación a género, sexualidad, clase social, discapacidad y raza. Por discursos esencialistas aprendidos en la escuela se entiende aquellas prácticas discursivas que "tienden a establecer vínculos causales entre identidades y las experiencias sociales o comportamientos de las personas" (Rasmussen, 2004, p. 63). Junto con esta esencialización, la jerarquización de estas identidades naturalizadas provoca que existan lugares epistémicos superiores y otros subordinados. Esto se refiere a que no todos/as tienen la autoridad para nombrar las identidades que se toleran y respetan, lo que tiene como efectos prácticas discriminatorias de abuso físico y emocional.

La caracterización de esta reproducción cultural de la discriminación en Chile que permite hacerse desde las estadísticas, es la siguiente. En la última encuesta UNICEF (2011) en la que se encuesta a niños y niñas, jóvenes de $7^{\circ}$ a $4^{\circ}$ año medio en relación a temas de discriminación y prejuicios, se concluye que los adultos son los que más discriminan en la escuela. También, se indica que el mayor grado de prejuicio se expresa hacia la homosexualidad y a personas con VIH/SIDA y que se mantienen los prejuicios sobre género y etnia entre las encuestas de los años 2004 y 2011. Junto con este tipo de datos, la encuesta CEP (2012), que indaga por conceptos de mujer, familia y trabajo, también entrega información relevante para entender las formas de razonar los roles asignados al género que se reproducen en la sociedad chilena. Por ejemplo, frente a la pregunta de quién debiera organizar ciertas actividades de la casa, como preparar la comida, lavar la ropa, cuidar enfermos o limpiar la casa, entre el $58 \%$ y $77 \%$ de los encuestados piensan que estas debieran ser realizadas por mujeres.

Lo relevante de estas cifras es que este ordenamiento social y cultural se aprende en la escuela a través de diversos mecanismos, muchos de los cuales se orientan a enfatizar la dimensión actitudinal frente a la diferencia, la importancia de la convivencia sana entre pares y relaciones interpersonales, etc. Sin embargo, lo que esta investigación problematiza es, de qué manera y a través de qué mecanismos la escuela enseña a razonar estas formas de pensar las identidades, a ordenarlas en buenas/malas; mejores/peores o normales/raras, y cómo se aprende a privilegiar ciertas identidades por sobre otras. Si bien es cierto, muchos estudios destacan la influencia de los medios de comunicación, la publicidad, así como la familia y otras instituciones sociales en la producción de estas formaciones, lo que planteamos es que hay que explorar en aquello que se produce en la escuela, como ámbito de formación explícito, ya que si los y las estudiantes aprenden estas forma de razonar las identidades y sus relaciones, entonces es un tipo de conocimiento que se puede localizar y rastrear.

Por tanto, el problema de este proyecto no se sitúa en la forma tradicional de pensar cuáles son los/las estudiantes de diferentes contextos que están aprendiendo de manera diferente. Más bien, el problema que este proyecto plantea es que todos y todas las estudiantes están aprendiendo formas que perpetúan sistemas de razonamiento 
naturalizadores de identidades, comunidades y relaciones. Por lo tanto, planteamos que los supuestos que sostienen las prácticas y conocimientos discriminatorios son parte de una forma de conocer y que, por lo tanto, se aprenden (Britzman, 1992). Como consecuencia, se pueden desaprender o aprender de otra manera.

En referencia al segundo grupo de argumentos para fundamentar nuestras preocupaciones, se encuentran las nuevas políticas educativas que buscan regular los abusos y prácticas violentas dentro de la escuela con leyes que instalan normas de sanción y castigo (Ley sobre Violencia Escolar, $\mathrm{N}^{\circ}$ 20. 536, 2011;). Estas formas de enfrentar los temas de abusos y discriminación, por lo menos, provocan preguntarse por el tipo de trabajo que debiera hacerse antes de llegar al tema de la sanción y castigo. El abordar estos problemas de bullying y discriminación con prácticas orientadas a la tolerancia y al respeto "por el/la diferente" no modifican estructuras del cómo se razona la normalidad y la diferencia. Más bien, la tolerancia es una forma de regular la aversión hacia esta diferencia y, por lo mismo, genera otros tipos de discriminación. Lo anterior, puede verse sustentado con algunos estudios nacionales que indican que nuestra sociedad está transitando de formas manifiestas de expresión del prejuicio hacia otras más sutiles (Cárdenas \& Barrientos, 2008). Lo que se plantea como problema en esta investigación es que en la medida que no se enseñe un conocimiento que tenga que ver con los efectos del esencializar y naturalizar conocimientos, personas y comunidades, la naturalización o esencialización de la diferencia seguirá siendo el marco dominante para construir sujetos escolares. Estas prácticas más bien instalan la producción de sujetos marcados para ser tolerados, lo que para esta investigación se ha contemplado como parte del problema.

Los discursos de tolerancia posicionan a los sujetos marcados como desviados, marginales o indeseables por lo que están en la condición de ser tolerados, lo que inevitablemente trae consigo el ejercicio de una superioridad, no problematizada, por parte de aquellos/as que son entrenados/as para tolerar. Wendy Brown (2006) explica que los discursos de tolerancia promueven, simultáneamente, tres acciones: (1) intensifican los efectos reguladores de las identidades esencializadas; (2) constituyen una "diferencia" fuera de un sistema de subordinación o inequidad; y (3) fortalecen la hegemonía de la identidad no marcada o dominante. Por ejemplo, cuando los/as estudiantes de educación básica son invitados a tolerar la raza, etnia, cultura, religión, orientación sexual de otros/ as, no hay ninguna sugerencia de cómo estas diferencias o identidades son producidas, de cómo han sido histórica y culturalmente construidas y, menos aún, mostrar que ellas mismas son el efecto del poder y de normas sociales y culturales incuestionadas. Más bien, la diferencia misma es la que los/las estudiantes aprenden a tolerar (Brown, 2006). Las lógicas de sanción y castigo, más los discursos instalados de tolerancia y respeto por la "diferencia" que hoy ocupan buena parte de los discursos oficiales de los establecimientos educativos, promueven formas de organizar la diferencia y la normalidad que reproducen sistemas desiguales.

Nuestro supuesto es que la diferencia, al continuar siendo presentada y estudiada como un algo que es, como un fenómeno en sí, como una esencia, restringe las posibilidades de modificar aquellas estructuras sociales y culturales que producen sistemas inequitativos. Al plantear que la diferencia solo es posible de ser reconocida, nombrada y descrita en función de una normalidad instalada, nos da posibilidad de ampliar nuestro campo de exploración para sugerir propuestas de cambio que tienen un impacto más concreto y transformador. Es desde aquí que proponemos una forma de investigar en la escuela. 


\section{LA PRODUCCIÓN DE LA NORMALIDAD EN LA ESCUELA}

Generar conocimiento acerca de cómo se produce, reproduce y circula la normalidad en la escuela es un desafío metodológico. En nuestro caso particular debíamos someter a cuestionamiento las formas tradicionales en que se documentan temas de diversidad e inclusión en la escuela para poder generar un conocimiento que nos permitiera contestar nuestras preguntas de investigación. En esta sección puntualizaremos tres aspectos del diseño metodológico, asociados a dispositivos metodológicos específicos, a través de los cuales hacerse cargo de la complicidad entre lo político y lo epistemológico que hemos asumido en la construcción del problema que anima la investigación. No se pretende dar una descripción completa de la metodología proyectada y realizada, sino detenernos en aquellas decisiones gruesas que definen la estrategia de investigación.

Es así como, en primer lugar, consideramos que la etnografía era la mejor forma en que podíamos, por un lado politizar la acción y producción de la investigación, y por otro, tener acceso a cuestionar los supuestos con que se piensa el conocimiento y procedimientos que producen el conocimiento mismo. En otras palabras, la etnografía nos sirvió para sostener la idea de que el conocimiento es producido por el instrumento que se utiliza, que para los propósitos de nuestra investigación es vital de considerar para reconocer cómo las políticas culturales en general, y las políticas de investigación en particular, enmarcan la indagación. En segundo lugar, se dispuso de instancias de discusión interdisciplinaria en torno a los trabajos de campo, a lo largo de todo el proceso de la investigación. Estas instancias permitieron ir cuestionando la propia producción etnográfica a partir de la reflexión entre los etnógrafos y etnógrafas que realizan sus terrenos en diversas escuelas, así como entre investigadores que compartían el terreno en una escuela, además de reflexiones generadas en diálogo con investigadores de diversas disciplinas que escuchaban los avances etnográficos y los interrogaban desde posiciones analíticas diversas. En tercer lugar, sobre la base de los análisis que ya contenían una toma de distancia respecto de las observaciones etnográficas, se desarrolló una segunda fase del trabajo en terreno en las escuelas con una modalidad participativa que incluyó activamente a los actores de cada establecimiento, en un diálogo de aprendizaje y retroalimentación mutua, especialmente entre profesoras y profesores en ejercicio, e investigadores que trabajaron en una intervención de las prácticas escolares desde la experiencia singular de cada escuela.

En términos concretos, en nuestra investigación trabajamos con seis establecimientos de la comuna de Santiago. Estas escuelas variaban en su composición, administración, número de estudiantes, género de los y las estudiantes, adscripción a credos, etc. Dicho de otra manera, intentamos buscar aquellos contextos que representaran la mayor variación posible, con el fin de poder sostener la idea de que no importa el lugar donde se mire la producción de la normalidad, siempre está disponible para ser puesta en cuestión. La normalidad, como ideología, siempre se sostiene en algún supuesto que es posible advertir y narrar desde la investigación. En cada una de las escuelas participó un par de etnógrafos y etnógrafas que estuvieron ocho meses desarrollando una relación con los y las profesores y profesoras, directivos y estudiantes durante al año 2013. Estas investigadoras e investigadores produjeron textos etnográficos a través de los cuales se intentaba mostrar las intensidades, regularidades y disonancias presentadas en los contextos particulares. Específicamente, el foco de nuestras etnografías fue interrogar las formas tradicionales en que los circuitos de las vidas particulares, las experiencias escolares, las jerarquías 
culturales y las relaciones de poder se expresan en los relatos etnográficos de las escuelas, en particular a través de las nociones estables de las identidades. Nuestra reflexión, una vez avanzada la producción etnográfica, se dirigió a que las formas tradicionales de entender y narrar la escuela requieren que sostengamos ideas e imaginaciones de unas identidades reconocibles, especialmente a través de la repetición de binarismos de género, raza, clase social, habilidad y edad para la producción de sujetos escolares. Este reconocimiento de posiciones específicas para las identidades en los contextos escolares implica pensar acerca de raza, sexualidad, género, habilidad y edad, como tecnologías que mapean las relaciones de los sujetos, las disposiciones de los agentes escolares y mantiene divisiones, prácticas y significados pensados e imaginados como naturales.

Por ello, en segundo lugar, fue necesario redirigir el foco de atención desde el registro de prácticas inequitativas individuales en las escuelas, a las operaciones del poder en las representaciones e ideas que reproducimos cuando generamos notas etnográficas, que buscan documentar la normalidad de la vida cotidiana escolar. Trabajar en seis contextos diferentes produciendo textos etnográficos en relación a la producción de la normalidad y la diferencia, nos ayudó a cuestionar las estabilidades del lenguaje disponible en la etnografía para nombrar y describir categorías, las operaciones del poder en este nombrar y sus relaciones específicas en los contextos escolares. Sin embargo, este redireccionamiento de la pregunta requirió trascender la etnografía, poniéndola en constante relación y tensión con perspectivas de otros investigadores, menos compenetrados de la cotidianidad de estas escuelas. Pues al poco andar de la investigación, nos dimos cuenta de que las descripciones realizadas por los y las etnografías también debían ser sometidas a la misma pregunta de investigación inicial que guiaba la entrada de estos investigadores e investigadoras a los espacios escolares. Esto es esperable en la medida en que la investigación etnográfica consiste en el desarrollo de una sensibilidad y un conocimiento de una escena desde dentro, a partir de la habitualidad de las prácticas que conforman dicha escena. Ese es precisamente el valor de la etnografía que se requería en un primer plano de la operación investigativa, pero constituye una limitación en un segundo plano: ¿En qué medida, las mismas descripciones etnográficas no podían escapar de las matrices dominantes para hablar y documentar la producción del género, de la sexualidad, de la raza, de la clase social en los contextos escolares que estábamos observando? Dicho de otra manera, la misma etnografía y las formas de producir textos acerca de lo observado debía ser visto como una reproducción de la normalidad.

En este plano, si los supuestos esenciales de la producción de textos etnográficos no son sometidos a cuestionamiento, estaríamos generando información que ya conocíamos y, por lo tanto, no lograríamos llegar a cumplir los objetivos iniciales planteados por nuestra investigación. Por lo tanto, el estudio etnográfico debía quedar, desde su inicio, abierto a la crítica ideológica, por lo que se diseñó para irse haciendo mientras se hacía, con el fin de poder ir, paso a paso del terreno etnográfico, pensando y modificando el lenguaje que era usado para generar las preguntas al terreno y, en consecuencia, los documentos generados producto de la observación.

En otras palabras, la producción de lo пиevo del terreno se convirtió en una condición política y epistemológica de la investigación. El "describir experiencias en contextos escolares", hacer el esfuerzo de atender al acontecimiento, se tradujo en la forma de asegurar la articulación política frente a temas de normalidad y diferencia, vigilando permanentemente cómo los marcos culturales y sociales no tradicionales podían alterar de 
manera significativa y esperada aquello que estábamos llamando la experiencia escolar de la diferencia. Es así como el hábito predominante-desde la escuela a las políticas sociales y de ahí a las categorías de investigación- de nombrar sujetos, características y relaciones de poder de una manera normativa, pasó a ser nuestro espacio para politizar los alcances del cómo conocemos y los efectos de este conocer.

Se trata de hacer estallar la intimidad etnográfica mediante la sensibilidad crítica, hacerla operar en un "segundo nivel", o sea, de otro modo, abriendo la soledad de la observación a la luz pública de los discursos sociales. Este giro ha permitido que cinco equipos multidisciplinarios de investigadores desarrollen análisis específicos con diversas metodologías y focos temáticos. Los registros etnográficos volvieron a interpelarse una y otra vez desde conceptos e hipótesis alejadas del terreno. Dichos registros cobraron más y más vida. Pero la condición de esta riqueza hermenéutica ha sido abandonar la referencia a la realidad de la cotidianidad escolar y la experiencia escolar de la diferencia independientemente de las observaciones que fueron motivadas y debatidas a lo largo del terreno. El precio pagado por el cuestionamiento ideológico de nuestras propias observaciones ha sido cortar el lazo etnográfico que une la experiencia y la interpretación. Inversamente, la ganancia de este extrañamiento interno al proceso de investigación ha sido libertad para interpretar la realidad frente a cualquier pretensión de poseer la interpretación más válida de los hechos. Esta es la condición político-epistemológica del dispositivo analítico que continúa trabajando, con apoyo de los mismos investigadores de terreno, en entender cómo aprendemos y enseñamos la diferencia (y la normalidad) en las escuelas.

En tercer lugar, la producción crítica de la puesta en debate de las observaciones etnográficas, que por un lado ha alimentado un abundante proceso de análisis interpretativo acerca de la experiencia escolar de la diferencia, por otro lado conoce un límite en la conversación con las escuelas, sus actores y acontecimientos. El período de terreno etnográfico fue seguido de un año de un trabajo colaborativo en las escuelas, particularmente con profesores en ejercicio, de modo que el conocimiento generado ya no pertenecía a un observador sino a un conjunto de participantes -investigadores y actores escolares- de una actividad conjunta que buscaba al mismo tiempo generar cambios en las prácticas de los y las profesoras y generar conocimiento académico. La denominación de "intervención" para calificar este trabajo es insatisfactorio, pues precisamente lo que se ha buscado es co-producir un conocimiento que sea de utilidad para que profesores y profesoras puedan modificar voluntariamente sus prácticas, y una participación política que conformar un saber teórico-práctico, con la consiguiente co-autoría respecto del trabajo realizado. En este tercer nivel de la investigación, lo político y lo epistemológico volvieron a entremezclarse de otro modo. El análisis ideológico unilateral fue así sometido a un diálogo permanente con las nuevas experiencias escolares de la diferencia, afinando interpretaciones y abriendo nuevas preguntas.

Este trabajo se avanza con un proceso de "intervención" en las mismas escuelas en donde se desarrollaron las etnografías iniciales. Esta intervención, que para nuestra forma de nombrar fue un trabajo colaborativo y de acompañamiento con profesores y profesoras, significó poner a prueba aquello que se había producido en las etnografías y que debía ser devuelto a la comunidad escolar en donde este había sido producido. Una vez más, aquello producido como conocimiento etnográfico articulaba aquello que se nombra como experiencia escolar con la producción misma de este conocimiento. En términos concretos, esta fase de la investigación tuvo una duración de 6 meses de trabajo semanal con profesores 
y profesoras (en algunos casos más extensos) en donde la dinámica fue que una dupla de investigadores e investigadoras se reunía con los y las participantes para desarrollar un "taller" al mes y luego las tres semanas restantes de este mes una investigadora acompañaba a estos profesores y profesoras (6) participantes diariamente en sus actividades regulares produciendo registros con distintos propósitos. Esta actividad, al ser totalmente voluntaria, nos proveyó de un espacio de politización necesario para trabajar las temáticas levantadas en las etnografías particulares de cada escuela participante.

Este complejo proceso de investigación es y ha sido permanentemente sometido a la vigilancia política y epistemológica que requiere y exige nuestra pregunta de investigación. Sin duda alguna, un aprendizaje relevante y significativo a la hora de investigar la articulación entre normalidad y diferencia en la escuela.

\section{DISCUSIÓN}

En este artículo hemos expuesto la construcción del problema y su traducción a opciones metodológicas, dando cuenta de un proceso de investigación a propósito de las relaciones íntimas entre lo epistemológico y lo político, que se presentan tradicionalmente como el dilema entre conocimiento e interés, o entre ciencia y transformación social. Con el fin de dar cuenta de nuestra experiencia de investigación, nos ha interesado profundizar en cómo las dimensiones políticas y epistemológicas se informan mutuamente en el diseño mismo. Por ello, para cerrar se discuten algunos aspectos de lo que podría denominarse el "dilema político-epistemológico" en investigación social crítica.

Hablar de normalidad y diferencia en educación implica una forma de razonar y actuar desde donde se reconoce que la escuela produce inequidad social y cultural a través de la reproducción de ciertos ordenes sociales y culturales de manera permanente y, como consecuencia, tanto los y las profesoras en formación y en ejercicio deben conocer cómo estas formas de producir diferencia y normalidad operan, a través de qué mecanismos y cuáles son las posibilidades de las que disponemos para modificar estas estructuras. Esta es una pregunta profundamente política.

$\mathrm{Al}$ inicio de la presentación levantamos unas preguntas de investigación que proponen que la diferencia no es una construcción aislada ni natural, sino que es una producción que se realiza en función de una normalidad imaginada y sostenida por instituciones, discursos y prácticas específicas que se pueden rastrear para comprender sus efectos y operaciones. Las preguntas hacen referencia a un proceso ideológico, a la producción de las formas en que presentamos argumentos para definir y diagnosticar al desviado, al que opera fuera de las normas de lo aceptado, valorado y deseado. Por ello, reconocemos en las preguntas de investigación un primer carácter político, en tanto remiten a ideologías y formas de pensar "lo natural" y "lo obvio" de las posiciones sociales y culturales de los sujetos.

Luego se argumenta que para avanzar en un conocimiento crítico de la diferencia es imperativo que se ponga en la escena aquello en relación a lo cual valoramos algo como diferente, raro, extraño. La indagación científica se vuelve así política en un segundo sentido, en tanto cuestiona las condiciones de producción de las normalidades naturales y obvias.

En un tercer sentido se ha afirmado el carácter político de la investigación, en la medida en que la pregunta por la producción de la normalidad y la diferencia en la escuela obliga 
a cuestionar los supuestos en que se fundan las políticas públicas hacia la diversidad, que son a partir de las cuales se configuran los problemas escolares, y que tienen un impacto en cómo se organizan y priorizan los temas y formas de hacer investigación en estas áreas.

En su conjunto, estos tres sentidos de lo político en la investigación marcan los tres pasos con los cuales se cierra un círculo que ata lo político y lo epistemológico. Tradicionalmente, estas relaciones se plantean como un dilema entre la pureza epistemológica y el compromiso político, como si ambos fueran esfuerzos contrapuestos. Nuestra experiencia de diseño de una investigación, ya en curso desde hace tres años, permite sostener que se trata de vectores convergentes, a veces de manera que no requieren esfuerzos especiales para favorecer esta convergencia, sino la sola consciencia de las condiciones y los efectos del conocimiento social.

Cuando se plantea el cambio y la transformación de estructuras sociales y culturales, sin duda que ello nos lleva a un terreno donde hay temas que son más complejos y sensibles que otros. La producción de conocimiento siempre es política. Por esto, nuestra convicción está amparada en que si bien es cierto, los sistemas educativos actuales ponen presión en las escuelas para servir valores de competición y crecimiento económico, nosotros desde este proyecto insistimos en que los espacios educativos son lugares para la producción de conocimiento creativo y transformador, la promoción de la vida reflexiva y del bienestar público. Sin duda que esto reconfigura los ámbitos políticos de la investigación y de las instituciones.

\section{REFERENCIAS BIBIOGRÁFICAS}

Baez, B. (2004). The study of diversity: The "knowledge of difference" and the limits of science. The Journal of Higher Education, 75(3), 285-306.

Britzman, D. (1992). The terrible problem of knowing thyself: Toward a poststructural account of teacher identity. Journal of Curriculum Theorizing, 9(3), 23-46.

Brown, W. (2006). Regulating aversión. Tolerance in the Age of Identity and Empire. Princeton: Princeton University Press.

Cárdenas, M., \& Barrientos, J. (2008). Actitudes explícitas e implícitas hacia los hombres homosexuales en una muestra de estudiantes universitarios en Chile. Psykhe, 17(2), 17-25.

Centro de Estudios Públicos (1995). Estudio Nacional de Opinión Pública. Recuperado el desde http://www.cepchile.cl/dms/archivo_4844_2963/encuestaCEP_junio-julio1995_completa.pdf

Centro de Estudios Públicos (2012). Estudio Nacional de Opinión Pública. Recuperado desde http://www.cepchile.cl/dms/archivo_5110_3266/encuestaCEP_jul-ago2012.pdf

Davies, L. (2013). The End of normal. Identity in a biocultural era. Ann Arbor: The University of Michigan Press.

Infante, M. (2007, septiembre). Inclusión Educativa en el Cono Sur: Chile. Taller Regional Preparatorio sobre Educación Inclusiva América Latina, Regiones Andina y Cono Sur. Unesco, Buenos Aires, Argentina.

Infante, M., \& Matus, C. (2009). Policies and practices of diversity: reimagining possibilities for new discourses. Disabilitity \& Society, 24(4), 437-445.

Ley Sobre Violencia Escolar N²0. 536 (2011). Sobre violencia escolar. Biblioteca del Congreso Nacional. Recuperado desde http://www.leychile.cl/Navegar?idNorma=1030087

Ley de Antidiscriminación $\mathrm{N}^{\circ} 20.609$ (2012). Establece medidas contra la discriminación. Santiago: Biblioteca del Congreso Nacional. Recuperado desde http://www.leychile.cl/ Navegar?idNorma $=1042092$ 
Ley de Inclusión Escolar $N^{\circ} 20.845$ (2015). De inclusión escolar que regula la admisión de los y las estudiantes, elimina el financiamiento compartido y prohíbe el lucro en establecimientos educacionales que reciben aportes del estado. Santiago: Biblioteca del Congreso Nacional. Recuperado desde http://www.mineduc.cl/usuarios/mineduc/doc/201501071223230. IndicacionesLeydeInclusionSenado.pdf

Matus, C., \& Infante, M. (2011). Undoing diversity: knowledge and neoliberal discourses in colleges of education. Discourse: Studies in the Cultural Politics of Education, 32(3), 294-330.

Organización de los Estados Americanos (OEA) (1998). Convención interamericana para la eliminación de todas las formas de discriminación contra las personas con discapacidad. Washington DC, USA.: Author.

Organization for Economic Co-operation and Development (OECD) (2008). Centre for educational research and innovation (CERI). Author. Recuperado desde http://www.oecd.org/ dataoecd/38/18/38446790.pdf

Rasmussen, M. L. (2004). “That's so gay!”: A study of the deployment of signifiers of sexual and gender identity in secondary school settings in Australia and the United States. Social Semiotics, 14(3), 289-308.en texto aparece el autor con una publicación en 2006

UNESCO (1994). Declaración de Salamanca y marco de acción sobre necesidades educativas especiales. Conferencia Mundial sobre Necesidades Educativas Especiales: Acceso y Calidad. Salamanca, España: Organización de las Naciones Unidas para la Educación, la Ciencia y la Cultura. Ministerio de Educación y Ciencia España.

UNESCO (2005). Guidelines for inclusion: Ensuring access to education for all. Paris: Author.

UNICEF (2011). La voz de los niños, niñas y jóvenes. Discriminación. Author. Recuperado desde http://unicef.cl/web/la-voz-de-los-ninos-ninas-y-adolescentes-y-discriminacion/ 